\title{
Rebound nystagmus: EOG analysis of a case with a floccular tumour
}

\author{
ATSUMI YAMAZAKI ${ }^{1}$ AND DAVID S. ZEE ${ }^{2}$ \\ From the ${ }^{1}$ Kozawa Eye Hospital, 2-2-11, Goken-cho, Mito-shi, Ibaragi, Japan, and the \\ ${ }^{2}$ Neurology Department, Johns Hopkins Hospital, Baltimore, Maryland, USA
}

SUMMARY Eye movements were recorded and quantitatively analysed in a patient with a tumour initially involving the cerebellar flocculus. Ocular motor abnormalities included (1) impaired smooth pursuit, (2) impaired cancellation of the vestibulo-ocular reflex when fixating an object rotating with the head, and (3) gaze paretic and rebound nystagmus. Comparable findings have been reported in monkeys with experimental floccular lesions. The rebound nystagmus (but not the other ocular motor abnormalities) disappeared when the tumour appeared to invade the brain stem in the region near the vestibular nuclei. This finding suggests that the floccular lesion unmasked a bias which created rebound nystagmus and that the bias probably arose in the vestibular nuclei.

Rebound nystagmus refers to a transient nystagmus that occurs when a patient returns the eye to the primary position after a prolonged attempt at eccentric gaze. Slow phases are in the direction of prior attempted eccentric gaze. It has been suggested that rebound nystagmus reflects a transient bias that has been created by the central nervous system in an attempt to hold eccentric gaze (Hood et al., 1973; Zee et al., 1976). Rebound nystagmus occurs even in normal individuals if, after a prolonged attempt at holding eccentric gaze, they return their eyes to the primary position in darkness (Zee et al., 1976). Darkness prevents the visual stabilisation systems from nulling the bias that causes rebound nystagmus. Therefore the bias of rebound nystagmus may be a normal compensatory phenomenon. In patients then rebound nystagmus appears in the light as well, because their visual stabilisation systems (that is, smooth pursuit) are impaired.

Hood et al. (1973) described rebound nystagmus as a useful sign of disorders of the cerebellum. However, it is not yet known where the bias of rebound nystagmus is created (Sharpe, 1974). Recently we observed a patient with a presumed floccular-nodular lesion who had gaze paretic and rebound nystagmus. However, the rebound but not the gaze paretic nystagmus disappeared when the tumour extended into the brain stem in the region of the root entry zone of the vestibular nerve, brachium pontis, and vestibular nuclei. We report a

Correspondence to Dr A. Yamazaki. detailed oculographic analysis of this patient's eye movements before and after several operations.

\section{Methods}

Electro-oculograms (EOG) of horizontal eye movements were recorded with a high-impedance direct current amplifier coupled to silver-silver chloride electrodes fixed at points lateral to both outer canthi. The eye position signal was also electronically differentiated to obtain eye velocity. Analogue signals were displayed on a rectilinear pen writer. The overall band width of the recording system was $30 \mathrm{~Hz}$.

The ocular motor examination consisted of the following. (1) Fixation behaviour: fixation in the primary position, eccentric fixation, and fixation in primary position after a prolonged attempt at holding eccentric gaze (to elicit rebound nystagmus). (2) Smooth pursuit: pursuit gain (smooth eye velocity /target velocity) was measured as the eye passed through the primary position while the patient tracked a small target moving in a triangular wave fashion. (3) Vestibulo-ocular responses: vestibular nystagmus was elicited by rotating the patient en bloc in a Bárány chair in complete darkness. Mental arithmetic was used to maintain a constant mental state. Cancellation of the vestibulo-ocular reflex (VOR) was measured as the patient attempted to fixate a target rotating with her. Standardised hot and cold water caloric tests were performed with eyes open behind +30 lenses. The maximum 
slow phase velocity and duration of the induced nystagmus was calculated. Canal paresis was defined by the following formula: If $\left(30^{\circ} \mathrm{C}\right.$ left + $44^{\circ} \mathrm{C}$ left $)-\left(30^{\circ} \mathrm{C}\right.$ right $+44^{\circ} \mathrm{C}$ right $)$ was greater than 40 seconds a canal paresis was indicated. (4) Saccades: saccadic eye movements were measured during tracking of a target jumping in a nonpredictable stepwise fashion.

\section{Case report}

A 17-year-old woman came to the Department of Ophthalmology with the complaint of oscillopsia of 1 week's duration. She also had a 10-month history of headaches. Neurological examination showed questionable papilloedema but apart from the abnormalities of eye movement no other neurological findings. On clinical ocular motor examination she showed striking gaze paretic and rebound nystagmus as well as abnormal smooth pursuit. Fig. 1 shows a horizontal EOG recording of her nystagmus. In the primary position fixation was steady. On assuming eccentric gaze in either horizontal direction she showed gaze paretic nystagmus with a slightly decreasing slow-phase velocity wave form (Holmes, 1917; Zee et al., 1976), which diminished as she maintained eccentric fixation. The initial slow-phase velocity of gaze paretic nystagmus during attempted rightward fixation was higher than that during attempted leftward fixation. On return to the primary position rebound nystagmus appeared.

Fig. 2 shows the patient tracking a target moving in a triangular wave fashion. Smooth pursuit movements were bilaterally impaired necessitating catch-up saccades. However, the gain (smooth eye velocity/target velocity) for rightward tracking was less $(0 \cdot 21)$ than that for leftward tracking $(0 \cdot 71)$.

Fig. 3 shows the patient attempting to cancel her vestibulo-ocular reflex using a small fixation target
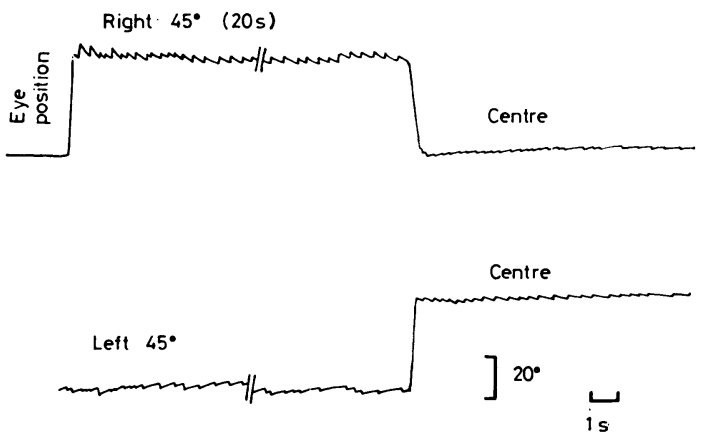

Fig. 1 Gaze paretic and rebound nystagmus prior to operation. Note the reversal of the direction of the nystagmus on returning to the primary position

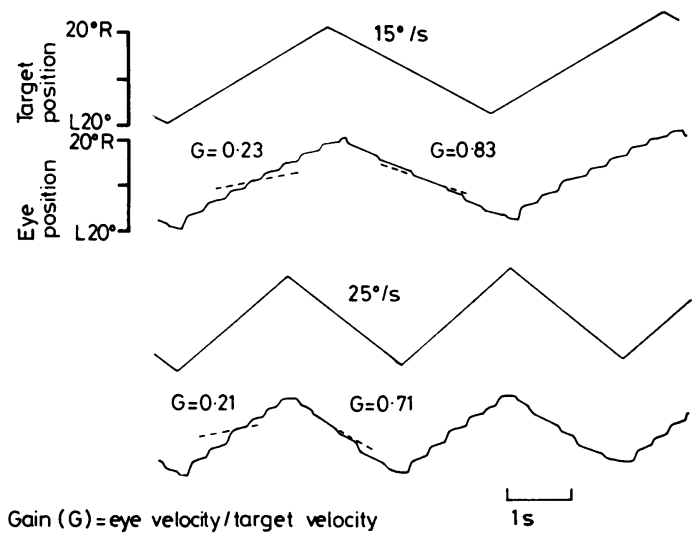

Fig. 2 Bilateral abnormality of smooth pursuit movements before operation. Pursuit gain showed asymmetric disturbance (rightward gains were less than leftward gains)

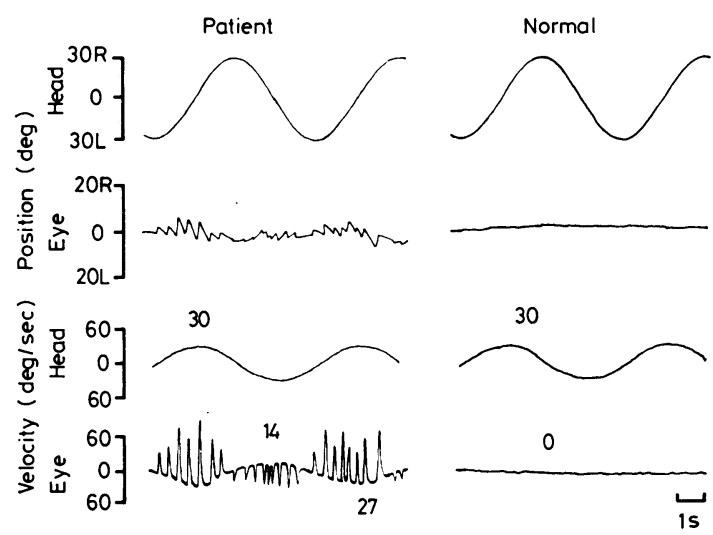

Fig. 3 Left tracing shows impaired cancellation of the vestibulo-ocular reflex by means of a fixation target moving with the head. The velocities of slow phases of nystagmus were $14^{\circ} / \mathrm{s}$ rightward and $27^{\circ} / \mathrm{s}$ leftward for a maximum velocity of $30^{\circ} \mathrm{s}$ for the head. Right tracking shows complete cancellation in a normal subject.

rotating with her head. Normally cancellation is complete and fixation is steadily maintained. However, the patient was unable completely to cancel her vestibulo-ocular reflex, so the eyes were taken off target by the vestibular slow phase requiring her to make saccades in order to reacquire the target. Cancellation during rightward head rotation was more severely affected (gain (eye velocity/head velocity) $=0.53$ ) than during leftward head rotation (gain $=0 \cdot 10$ ).

Caloric testing showed normal responses in both ears. Saccadic eye movements showed normal accuracy and velocity-amplitude relationships. 


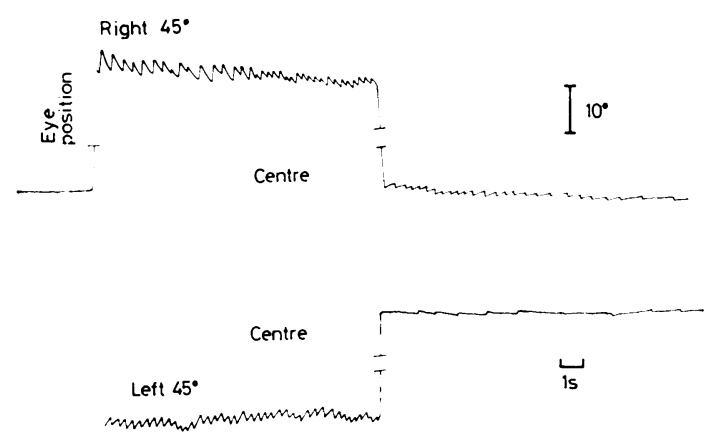

Fig. 4 Gaze paretic and rebound nystagmus after the first operation in which a tumour involving primarily the right vestibulocerebellum was removed. When gaze was directed towards the side of tumour (to the right), there was a large-amplitude gaze paretic nystagmus, whereas on gaze left a small-amplitude nystagmus appeared. Note the differences in duration and amplitude of nystagmus between right and left gaze

Neuroradiological studies suggested a mass in the posterior fossa, and the patient underwent a suboccipital craniotomy. A choroid plexus papilloma was found in the fourth ventricle involving the right flocculus, nodulus, part of the right cerebellar hemisphere, and a part of the left flocculus as well. The tumour was partly removed together with a portion of the right flocculus and paraflocculus.

After this operation the pursuit deficit and gaze paretic and rebound nystagmus persisted (Fig. 4). However, the abnormalities were more marked, especially on rightward gaze.

Two months later, just before reoperation in an attempt to remove the remaining part of the tumour, the patient was again examined. Gaze paretic nystagmus and impaired pursuit were still present. However, she no longer had rebound nystagmus. At the second operation residual tumour was found in the region of the right flocculus and nodulus as well as involving the brain stem at the juncture of the right vestibular nerve and brachium pontis. As much tumour as possible was removed.

Fig. 5 shows an EOG recording 20 days after the second operation. In the primary position there was a small-amplitude jerk nystagmus with slow phases to the right. This increased on gaze left. In addition on rightward gaze there was prominent gaze paretic nystagmus. This combination of gaze paretic nystagmus to the right and vestibular nystagmus to the left is so-called Bruns nystagmus (Bruns, 1908; Cushing, 1921). On return to the primary position after prolonged eccentric gaze there was no change in the slow-phase velocity of the primary position jerk nystagmus. In other words rebound nystagmus was no longer present.
At this time caloric testing showed a definite canal paresis on the right side but a normal response on the left side. Fig. 6 shows an EOG recording of the cold water caloric-induced nystagmus. The maximum slow-phase velocity of nystagmus and the duration of responses showed a clear asymmetry, that is, a canal paresis on the right side. Saccadic eye movements were accurate and had a normal velocity-amplitude relationship.

One year after the second operation the patient was re-examined. Now there was no primary-

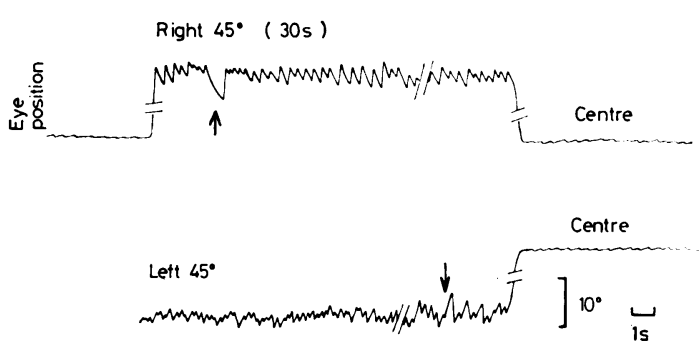

Fig. 5 Recording of horizontal eye movements after second operation in which the tumour was found to involve the brain stem. In the primary position a smallamplitude jerk nystagmus with slow phases to the right is present. When gaze was directed towards the side of the tumour (right), there was a large-amplitude nystagmus showing an exponentially decaying centripetal drift velocity ( $\uparrow)$; on gaze left a small-amplitude, rapid nystagmus exhibiting slow phase of constant velocity $(\downarrow)$ appeared. On return to the primary position no change in velocity of the primary-position nystagmus was noted
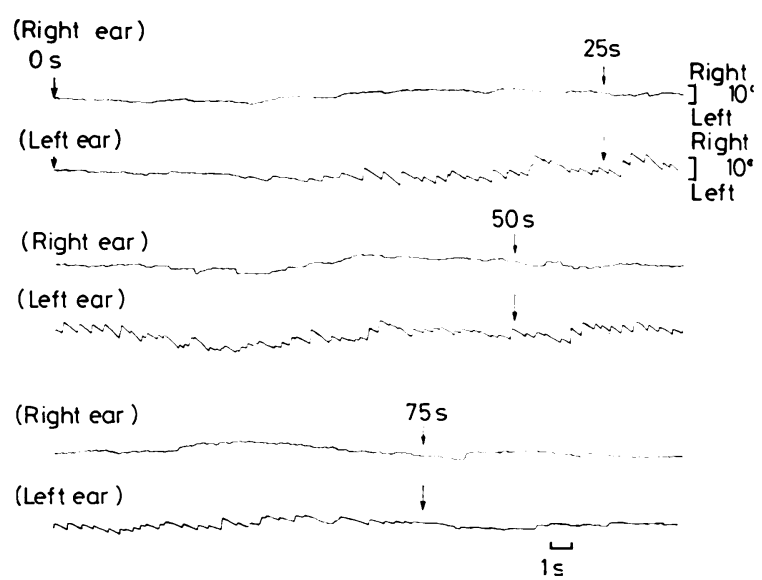

Fig. 6 Recording of $30^{\circ} \mathrm{C}$ water caloric-induced nystagmus after the second operation. The maximum slow-phase velocity and duration of the induced nystagmus by the right-ear stimulus were significantly decreased compared with the induced nystagmus by the left-ear stimulus 
position jerk nystagmus, though on caloric testing the right canal paresis persisted. Gaze paretic nystagmus and the smooth pursuit deficit were still present, and rebound nystagmus had reappeared. There were no signs of tumour recurrence.

\section{Discussion}

Our findings suggest that many of our patient's ocular motor abnormalities-gaze paretic and rebound nystagmus, impaired smooth pursuit, and cancellation of the vestibulo-ocular reflex-were related to tumour involvement of the cerebellar flocculus and possibly nodulus. In fact the constellation of ocular motor abnormalities shown by our patient has been reproduced in monkeys with bilateral floccular ablations (Zee et al., 1978). However, our patient's rebound nystagmus (but not the other ocular motor findings) disappeared when the brain stem and in particular the region of the vestibular nuclei, eighth nerve, and brachium pontis appeared to be involved. This finding suggests that the bias that creates rebound nystagmus is developed in the brain stem and not in the flocculus. In fact, the floccular lesion, by causing gaze paretic nystagmus and impairing smooth pursuit, acted as the stimulus to create the need for the production of a bias to counteract the centripetal drift of gaze paretic nystagmus. This bias manifests itself as rebound nystagmus. Of course other extra-floccular, cerebellar structures might be essential for the normal function of the brain stem network that creates the bias of rebound nystagmus.

One might next ask by what mechanism the bias of rebound nystagmus is created. Normally after a saccade the eye is held in its new position by the tonic output of a brain stem neural network that provides the appropriate position coded information for the ocular motor neurons to hold positions of gaze. This gaze-holding neural network has been termed a neural integrator, since functionally it mathematically integrates velocity into positioncoded information (Robinson, 1974). There are presumably 2 horizontal neural integrators (one for rightward and the other for leftward movements) that work reciprocally in a push-pull fashion. If the neural integrators become defective, eccentric gaze cannot be held, and the eye drifts back toward the primary position with an exponentially decaying velocity. In an attempt to maintain eccentric fixation repetitive eccentric saccades are required, resulting in the pattern of gaze paretic nystagmus. However, if the defect is symmetrical, a null region exists near the primary position of gaze where fixation is steady.

The centripetal drift of gaze paretic nystagmus causes movement of images across the retina which impairs visual acuity. What adaptive mechanisms are available to the central nervous system to null gaze paretic nystagmus so as to improve visual acuity?

The smooth pursuit system could be used to detect the movement of images on the retina caused by centripetal drift and then promptly generate a motor command in an attempt to track the moving images. This tracking command would be oppositely directed to the direction of eye drift and would in effect null the gaze paretic nystagmus and permit steady fixation. However, in our patient and in most other patients with rebound nystagmus the smooth pursuit system is impaired. Therefore other somewhat slower acting mechanisms must be called upon in an attempt to hold the eyes steady.

Exactly how such mechanisms work or what stimuli bring them into play are not known. The central nervous system might detect the velocity of the continuous centripetal drift of gaze paretic nystagmus by using visual information about retinal slip and/or extraretinal information about eye motion. This stimulus could then be used to produce an oppositely directed velocity bias in an attempt to null the drift. Alternatively, the steady state position of the eyes might be monitored and after a prolonged attempt at holding eccentric gaze a position bias could be created to shift the null point of the 2 neural integrators towards the direction of attempted gaze holding. Exactly which of these mechanisms are operative in producing rebound nystagmus has not yet been established.

Finally, one must ask why our patient's rebound nystagmus reappeared 1 year after her last operation. Coincident with the return of rebound nystagmus the primary-position jerk nystagmus, presumably of vestibular origin, disappeared even though the caloric responses still showed a canal paresis. One can speculate that the central nervous system's repair mechanisms that rebalanced the vestibular nuclei to eliminate primary-position nystagmus also restored the ability of the brain stem to generate the bias for rebound nystagmus. In fact Haddad et al. (1978) have shown that repair of nystagmus after unilateral vestibular nerve lesions can be accomplished without a vestibulocerebellum. However, the reappearance of rebound nystagmus may have simply been coincidental and related to other unknown mechanisms.

\section{References}

Bruns, L. (1908). Die Geschwulste des Nervensystems. Karger: Berlin.

Cushing, H. (1921). Further considering the acoustic neuromas. Laryngoscope, 31, 209-228.

Haddad, G. M., Friendlich, A. R., and Robinson, D. A. 
(1978). Compensation of nystagmus after eight nerve lesions in vestibulocerebellectomized cats. Brain Research, 135, $192-196$.

Holmes, G. (1917). Disturbances of the ocular movements and nystagmus. Brain, 40, 500-505.

Hood, J. D., Kayan, A., and Leech, J. (1973). Rebound nystagmus. Brain, 96, 507-526.

Robinson, D. A. (1974). The effect of cerebellectomy on the cat's vestibulo-ocular integrator. Brain Research, 71, 195-207.
Sharpe, J. A. (1974). Rebound nystagmus-A cerebellar sign?, Journal of the American Medical Association, 227, 648-649.

Zee, D. S., Yamazaki, A., and Gucer, G. (1978). Ocular motor abnormalities after flocculectomy in trained monkeys (Abstract), Investigative Ophthalmology, 17, Suppl. 217.

Zee, D. S., Yee, R. D., Cogan, D. G., Robinson, D. A., and Engel, W. K. (1976). Ocular motor abnormalities in hereditary cerebellar ataxia. Brain, 99, 207-234. 